\title{
The N-terminus of Lactobacillus amylovorus feruloyl esterase plays an important role in its secretion by Lactobacillus plantarum and Escherichia coli
}

\author{
Zhenshang $\mathrm{Xu}^{1,2}$, Rongling Zhang ${ }^{3}$, Ting Wang ${ }^{1,2^{*}}$ and Jian Kong ${ }^{3^{*}}$ (1)
}

\begin{abstract}
Background: Feruloyl esterase is a multifunctional esterase with potential industrial applications. In the present study, we found the Lactobacillus amylovorus feruloyl esterase (FaeLam) could be secreted by L. plantarum and Escherichia coli. However, no signal peptide was detected in this protein as predicted by SignalP-5.0. Therefore, experiments were carried out to propose an explanation for the extracellular release of FaeLam.
\end{abstract}

Results: Here, we identified that the FaeLam could be secreted to the culture medium of L. plantarum CGMCC6888 and E. coli DH5a, respectively. To exclude the possibility that FaeLam secretion was caused by its hydrolytic activity on the cell membrane, the inactive FaeLam ${ }^{\text {S106A }}$ was constructed and it could still be secreted out of L. plantarum and E. coli cells. Furthermore, the truncated version of the FaeLam without the $\mathrm{N}$-terminal residues was constructed and demonstrated the importance of the 20 amino acids of N-terminus (N20) on FaeLam secretion. In addition, fusion of heterologous proteins with N20 or FaeLam could carry the target protein out of the cells. These results indicated the $\mathrm{N}$-terminus of FaeLam played the key role in the export process.

Conclusions: We proved the N-terminus of L. amylovorus FaeLam plays an important role in its secretion by L. plantarum and E. coli. To our best knowledge, this is the first reported protein which can be secreted out of the cells of both Gram-positive and Gram-negative bacteria. Furthermore, the results of this study may provide a new method for protein secretion in L. plantarum and E. coli through fusion the target protein to N20 of FaeLam.

Keywords: Feruloyl esterase, Secretion, N-terminus

\section{Background}

Feruloyl esterases are a subclass of carboxylic acid esterases that hydrolyze the ester bond between hydroxycinnamic acids and sugars [1]. As important auxiliary

\footnotetext{
*Correspondence: tingwang_97@hotmail.com; kongjian@sdu.edu.cn 1 State Key Laboratory of Biobased Material and Green Papermaking, Qilu University of Technology, Shandong Academy of Science, No.3501, Daxue Road, Jinan 250353, People's Republic of China

${ }^{3}$ State Key Laboratory of Microbial Technology, Shandong University, No.72, Binhai Road, Qingdao 266237, Shandong, People's Republic of China
}

Full list of author information is available at the end of the article enzyme for plant biomass degradation, they have been widely used in fuel ethanol production, pulp and paper industries, and animal feed additives [2]. In recent years, feruloyl esterases have aroused interest because of their ability to release hydroxycinnamic acids from plant materials to exert their bioactivity directly. Many studies in vitro and in vivo have indicated that hydroxycinnamic acids (especially ferulic acid) can prevent the oxidation of low-density lipoprotein, inhibit the spread of tumors, and protect against certain chronic diseases such as coronary heart disease and some cancers [3]. original author(s) and the source, provide a link to the Creative Commons licence, and indicate if changes were made. The images or other third party material in this article are included in the article's Creative Commons licence, unless indicated otherwise in a credit line to the material. If material is not included in the article's Creative Commons licence and your intended use is not permitted by statutory regulation or exceeds the permitted use, you will need to obtain permission directly from the copyright holder. To view a copy of this licence, visit http://creativecommons.org/licenses/by/4.0/. The Creative Commons Public Domain Dedication waiver (http://creativeco mmons.org/publicdomain/zero/1.0/) applies to the data made available in this article, unless otherwise stated in a credit line to the data. 
Considering the importance in various applications, many feruloyl esterases have been found and isolated from a large number of fungal and bacterial sources [4]. Recently, more and more feruloyl esterases have been identified and characterized in different Lactobacillus species, which have generally recognized as safe (GRAS) status and long history of use in food applications [5]. Given the essential role of feruloyl esterase in the production of hydroxycinnamic acids, plus the GRAS status of Lactobacillus species, which therefore have less regulatory concerns, feruloyl esterases from probiotic Lactobacillus strains can be directly used for increased production of high-value hydroxycinnamates and ferulic acid from natural or synthetic carbon sources [6]. In general, Lactobacillus feruloyl esterases are heterologously expressed in host cells and then purified for applications. However, whether feruloyl esterase can be secreted by Lactobacillus strains is still unknown [6, 7].

The Gram-negative bacterium Escherichia coli is a commonly used cell factory for the production of feruloyl esterases, because it is the best characterized host with many available expression and regulation tools [8, 9]. However, the common laboratory strains of E. coli are poor secretors for proteins, resulting from the complex cell envelope with two layers [10]. Unexpectedly, our previous study showed that the high yield of feruloyl esterase (FaeLam derived from L. amylovorus) could be secreted from $E$. coli BL21(DE3) with the pET expression system [11]. Despite the several reports about secretory proteins in $E$. coli, there is no conclusive and universally secretion pathway identified so far $[12,13]$. A greater understanding of feruloyl esterase secretion has application significance in protein production or therapeutic purposes.

In this study, we reported that the feruloyl esterase FaeLam from $L$. amylovorus CGMCC11056 could be secreted from $L$. plantarum CGMCC6888 and E. coli DH $5 \alpha$ respectively when heterologously overexpressed by Lactobacillus/E. coli shuttle vector. In order to further explore this anomalous secretory phenomenon, the inactive mutant and truncated mutants of FaeLam was constructed to assess the activity or sequence requirements for secretion. Moreover, the ability of FaeLam and its $\mathrm{N}$-terminal sequence as carriers to export protein was also performed.

\section{Results}

\section{Construction of the shuttle vector pLP3804}

To investigate the expression and secretion of FaeLam and its mutants in L. plantarum and E. coli, a Lactobacillus/E. coli shuttle vector named pLP3804 was constructed. The detailed composition of the expression vector pLP3804 was presented in Fig. 1A. This vector had a promoter $\mathrm{P}_{\text {tuf }}$, which had been verified to

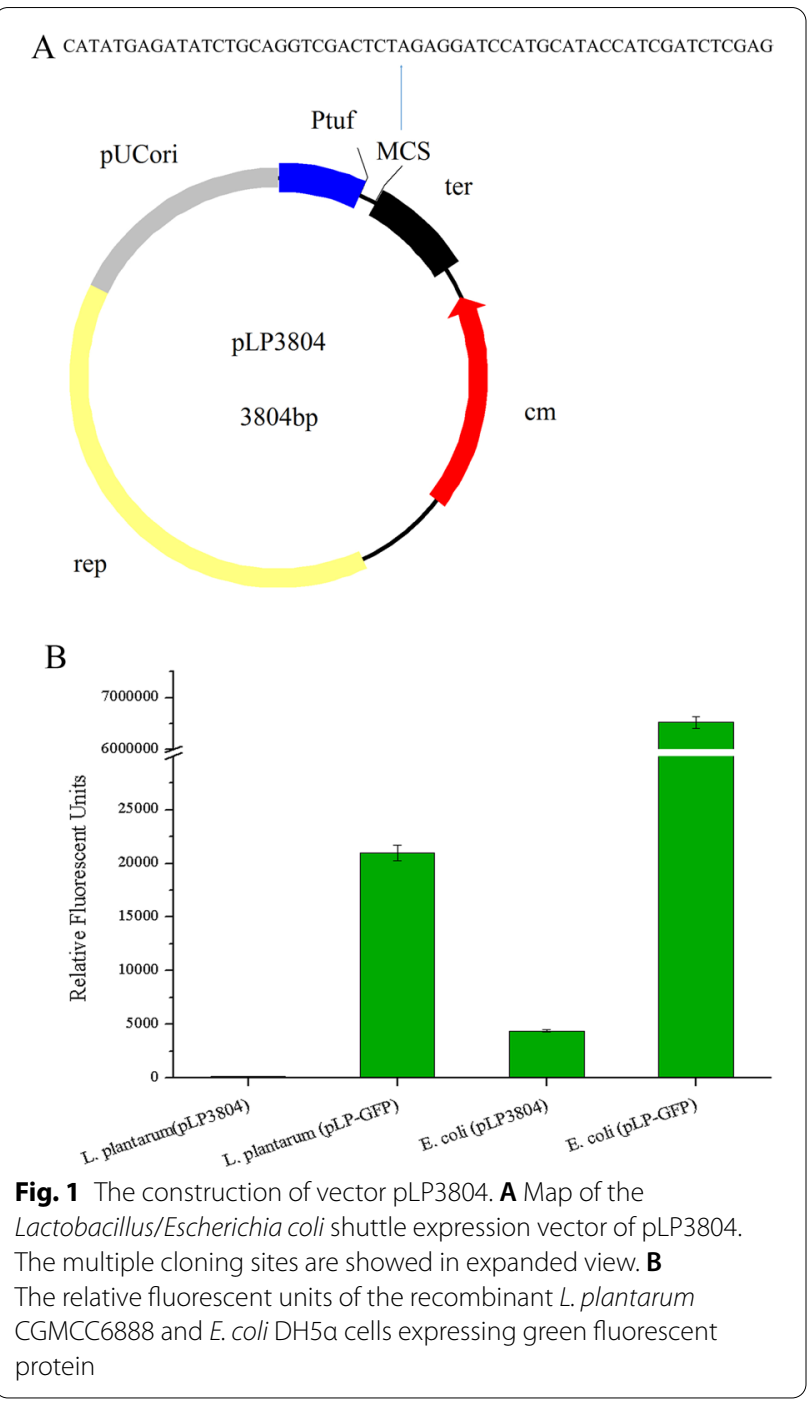

be able to express protein in L. plantarum constitutively [14]. Furthermore, the first restriction enzyme site contained in the multiple cloning sites was CAT ATG, which allowed the heterologous proteins to be expressed with a natural $\mathrm{N}$-terminus when restriction enzyme NdeI was applied. Green fluorescence protein (GFP) derived from vector pMN402 was used as the reporter protein to evaluate the feasibility of pLP3804 for heterologous protein expression in L. plantarum and E. coli [15]. The gfp gene was cloned into pLP3804 and the resultant plasmid pLP-GFP was transformed into competent cells of $L$. plantarum CGMCC6888 and E. coli $\mathrm{DH} 5 \alpha$. The green fluorescence in recombinant cells was observed (Fig. 1B), while no fluorescence was observed in control strains (L. plantarum CGMCC6888 and $E$. coli DH5 $\alpha$ with pLP3804), suggesting that the promoter $\mathrm{P}_{\text {tuf }}$ could initiate protein expression not 
only in L. plantarum but also in E. coli. SDS-PAGE was carried out to confirm the expression level of GFP. The results showed that one more protein band clearly appeared in the E. coli harboring pLP-GFP (Additional file 1: Figure S1). This phenomenon was consistent with the difference in fluorescence. Nevertheless, environmental conditions such as $\mathrm{pH}$ and dissolved oxygen also had negative effects on the fluorescence of GFP in L. plantarum [16].

\section{FaeLam is secreted by L. plantarum and E. coli}

\section{without typical signal peptide}

We previously reported that $L$. amylovorus CGMCCC11056 was capable to produce feruloyl esterase FaeLam, which consists of 247 amino acids with a molecular mass of $27.4 \mathrm{kDa}$ (accession number: AOR52353) [17]. Furthermore, no putative signal peptide motif was predicted at the N-terminal of FAE by using SignalP-5.0 server (http://www.cbs.dtu.dk/services/SignalP-5.0/) (Additional file 1: Figure S2A). The 744-bp of the faeLam gene was cloned from L. amylovorus CGMCC11056 and ligated into the $\mathrm{P}_{\text {tuf }}$-driven expression plasmid pLP3804, generating the pLP-FaeLam. This recombinant plasmid was transformed into L. plantarum CGMCC6888 and E.
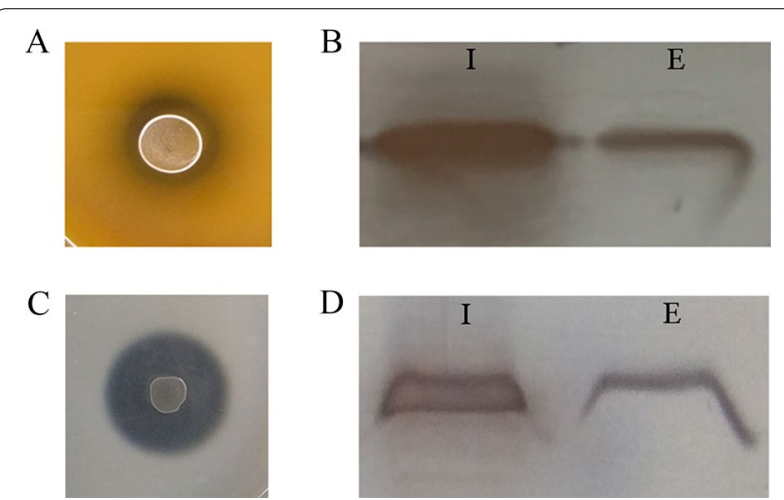

Fig. 2 Activity assay and subcellular localization of FaeLam. A $L$. plantarum CGMCC6888 containing pLP-FaeLam grown in MRS medium supplementary with ethyl ferulate; $\mathbf{B}$ Western blot analysis of the subcellular localization of FaeLam expressed in L. plantarum CGMCC6888. C E. coli DH5a containing pLP-FaeLam grown in LB medium supplementary with ethyl ferulate; $\mathbf{D}$ Western blot analysis of the subcellular localization of FaeLam expressed in E. coli DH5a. I: intracellular fraction; E: extracellular fraction coli DH5 $\alpha$ cells for overexpression. The recombinant cells could produce clear hydrolytic zone in the plates containing ethyl ferulate when incubation at $37^{\circ} \mathrm{C}$ for $12 \mathrm{~h}$, indicating that the FaeLam was correctly expressed (Fig. 2A, C). Furthermore, the distribution of FaeLam was investigated by detecting the activities of intracellular and extracellular fractions. As shown in Table 1, E. coli BL21(DE3) harboring pET22-FaeLam was used as a positive control. The extracellular $\mathrm{pH}$ drastically decreased when L. plantarum was cultured in MRS medium, resulting in a weak activity of FaeLam at acidic conditions. However, feruloyl esterase activity was detected in both intracellular and extracellular fractions of $L$. plantarum CGMCC6888 by HPLC (Additional file 1: Figure S3). Meanwhile, both of the fractions of $E$. coli DH5 $\alpha$ containing plasmid pLPFaeLam could produce hydrolytic zone according to the plate-based assay (Additional file 1: Figure S4).

The subcellular localization of FaeLam in L. plantarum CGMCC6888 and E. coli DH5 $\alpha$ was further determined by western blot. As shown in Fig. 2B and D, FaeLam protein bands of the same molecular weight were observed in both of the intracellular and extracellular fraction. All these results suggested that L. amylovorus CGMCCC11056 FaeLam could be exported into the medium when expressed in L. plantarum CGMCC6888 and E. coli DH5 $\alpha$. Analysis of the $\mathrm{N}$-terminal sequence of extracellular FaeLam of E. coli DH5 $\alpha$ showed that it was MSRITIERDGL, which matched the amino acid sequence of FaeLam from residues 1 to 11 (Additional file 1: Figure S5). This result proved that FaeLam was translocated without removing any $\mathrm{N}$-terminal sequence.

\section{Expression of inactive FaeLam}

$\mathrm{Su}$ et al. [12] reported that Thermobifida fusca cutinase could be secreted from cells when heterologously expressed in $E$. coli, which resulted from its hydrolytic activity toward phospholipids. To exclude the possibility that FaeLam secretion was caused by its hydrolytic activity on the cell membrane, the active site of FaeLam was mutated according to the results of amino acid sequence alignment with other feruloyl esterases. $L$. amylovorus FaeLam belongs to the $\alpha / \beta$-hydrolase family. The examination of FaeLam structure model reveals a Ser106-His225-Asp197 catalytic triad in which Ser106 is critical to the hydrolytic activity. Therefore,

Table 1 The intracellular and extracellular feruloyl esterase activities $(U / \mathrm{mL})$ in different recombinant strains

\begin{tabular}{llll}
\hline Feruloyl esterase activities & $\begin{array}{l}\text { L. plantarum CGMCC6888 } \\
\text { With pLP3804-FaeLam }\end{array}$ & $\begin{array}{l}\text { E. coli DH5a } \\
\text { With pLP3804-FaeLam }\end{array}$ & $\begin{array}{l}\text { E. coli BL21(DE3) } \\
\text { With pET22b-FaeLam }\end{array}$ \\
\hline Intracellular & $397.1 \pm 19.4$ & $1742.3 \pm 46.7$ & $3886.4 \pm 71.2$ \\
Extracellular & ND & $540.9 \pm 25.6$ & $2013.7 \pm 44.3$ \\
\hline
\end{tabular}

Feruloyl esterase activity was determined by using $\rho$ NPF as substrate. ND means no detectable activity via this method 
the site-directed mutagenesis of Ser106 to Ala was attempted. FaeLam ${ }^{\mathrm{S} 106 \mathrm{~A}}$ was obtained and the plasmid pLP-FaeLam ${ }^{\text {S106A }}$ was constructed. When the mutant was expressed in L. plantarum CGMCC6888 and E. coli DH5 $\alpha$, the feruloyl esterase activity of recombinant cells was not detected (Fig. 3A, C). However, the FaeLam ${ }^{\text {S106A }}$ protein band was still detected in both of the intracellular and extracellular fractions of L. plantarum CGMCC6888 and E. coli DH5 $\alpha$ (Fig. 3B, D).

\section{Expression of FaeLam with $\mathrm{N}$-terminal deletion}

Since the secretion of FaeLam was not due to the hydrolysis activity, whether the N-terminus of FaeLam played a role in its secretion was further investigated. The FaeLam protein mutant $(\Delta 20$ FaeLam) with 20 amino acid truncated at the $\mathrm{N}$-terminus was constructed. When E. coli $\mathrm{DH} 5 \alpha$ was transformed with pLP- $\Delta 20$ FaeLam, the recombinant cells could not produce hydrolytic zone in the plate (Fig. 4A), and the mutant protein was not detected in extracellular fraction by western blot (Fig. 4B). The same phenomenon was observed in $L$. plantarum CGMCC6888 harboring the plasmid pLP$\triangle 20$ FaeLam (data not shown). Furthermore, the SDSPAGE was performed to analysis the expression of FaeLam and $\Delta 20$ FaeLam in E. coli DH5 $\alpha$. As shown in Fig. $4 \mathrm{C}$, these two proteins were expressed with almost no difference as revealed in the whole cells analysis. However, the $\Delta 20$ FaeLam was not detected in the soluble fraction, suggesting it was deposited as inclusion bodies. Taken together, deletion of $\mathrm{N}$-terminus completely

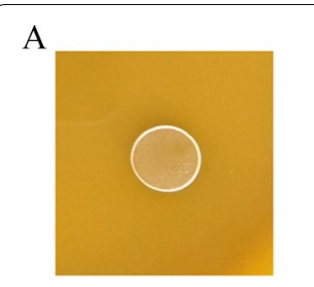

B

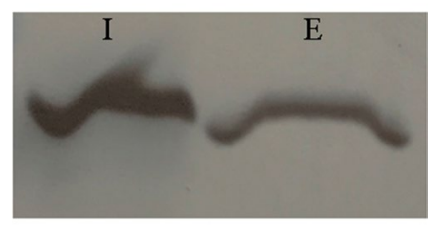

C

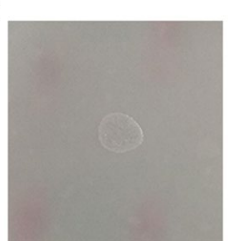

D



Fig. 3 Activity assay and subcellular localization of FaeLam ${ }^{5106 A}$. A $L$. plantarum CGMCC6888 containing pLP-FaeLam ${ }^{\text {S106A }}$ grown in MRS medium supplementary with ethyl ferulate; $\mathbf{B}$ Western blot analysis of the subcellular localization of FaeLam ${ }^{5106 \mathrm{~A}}$ expressed in L. plantarum CGMCC6888; C E. coli DH5a containing pLP-FaeLam ${ }^{\text {S106A }}$ grown in LB medium supplementary with ethyl ferulate; $\mathbf{D}$ Western blot analysis of the subcellular localization of FaeLam ${ }^{\text {S106A }}$ expressed in E. coli DH5a. I: intracellular fraction; E: extracellular fraction

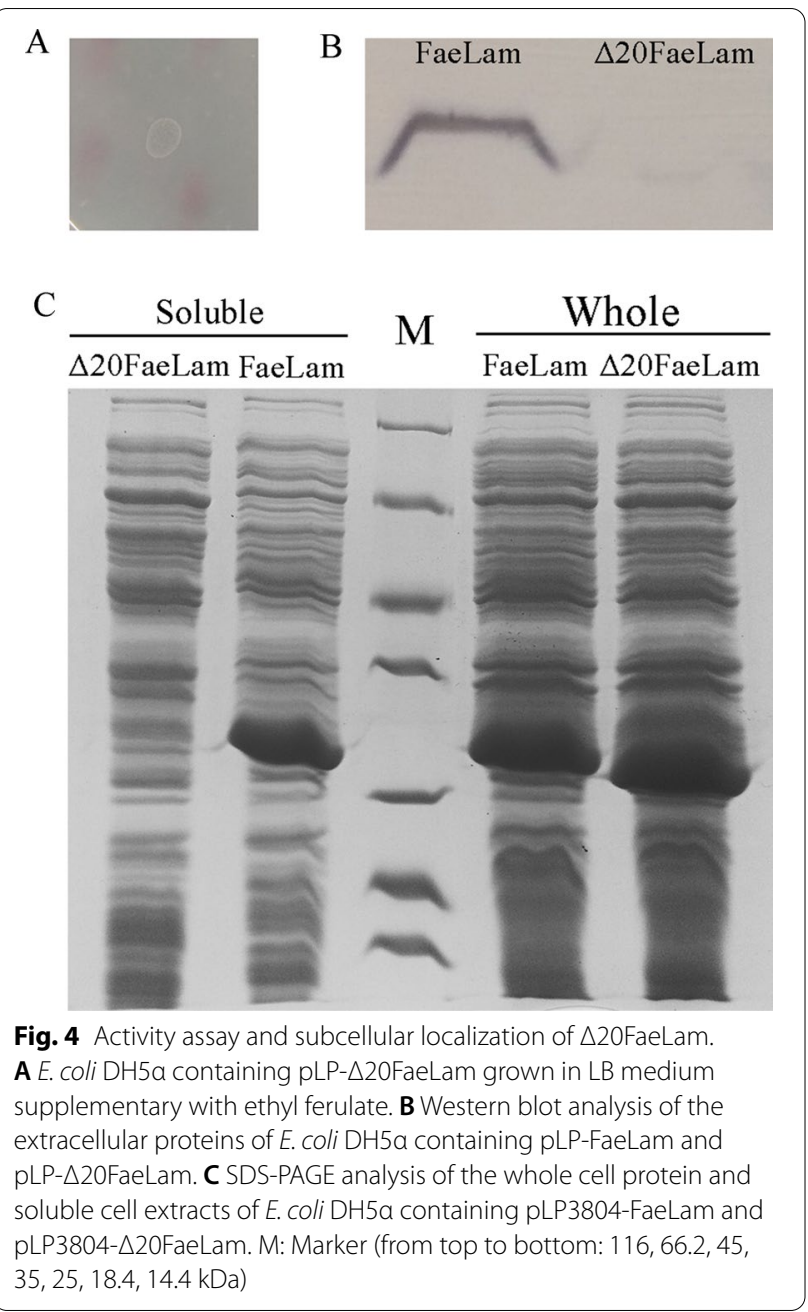

inhibited the formation of the soluble enzyme, indicating the N-terminal region of FaeLam might play an important role in its secretion like the Cel-CD [13].

\section{Secretion of heterologous protein by the $\mathrm{N}$-terminus}

To directly verify the $\mathrm{N}$-terminus sequence plays the key role in secretion, the $\mathrm{N}$-terminal 20 amino acid (N20) was used to guide the heterologous protein out of $L$. plantarum and E. coli. The carbohydrate binding module of xylanase (CBM, $14.2 \mathrm{kDa}$ ) from Paenibacillus panacisoli was selected and fused downstream to the N20, resulting of N20CBM [18]. As shown in the Fig. 5A and $\mathrm{B}$, this fusion protein were detected in the extracellular after $12 \mathrm{~h}$ cultivation of L. plantarum CGMCC6888 and E. coli DH5 $\alpha$. Furthermore, we also tested if the FaeLam could be used as a fusion partner to carry CBM out of the cells in recombinant $L$. plantarum and $E$. coli. The results showed that FaeLamCBM could also be secreted into the medium after $12 \mathrm{~h}$ cultivation. As the controls, the CBM can only be detected in the intracellular fraction when 

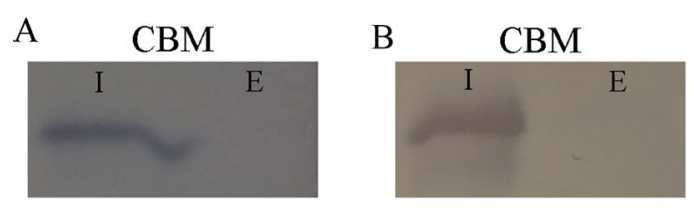

\section{N20CBM FaeLamCBM FaeLam}
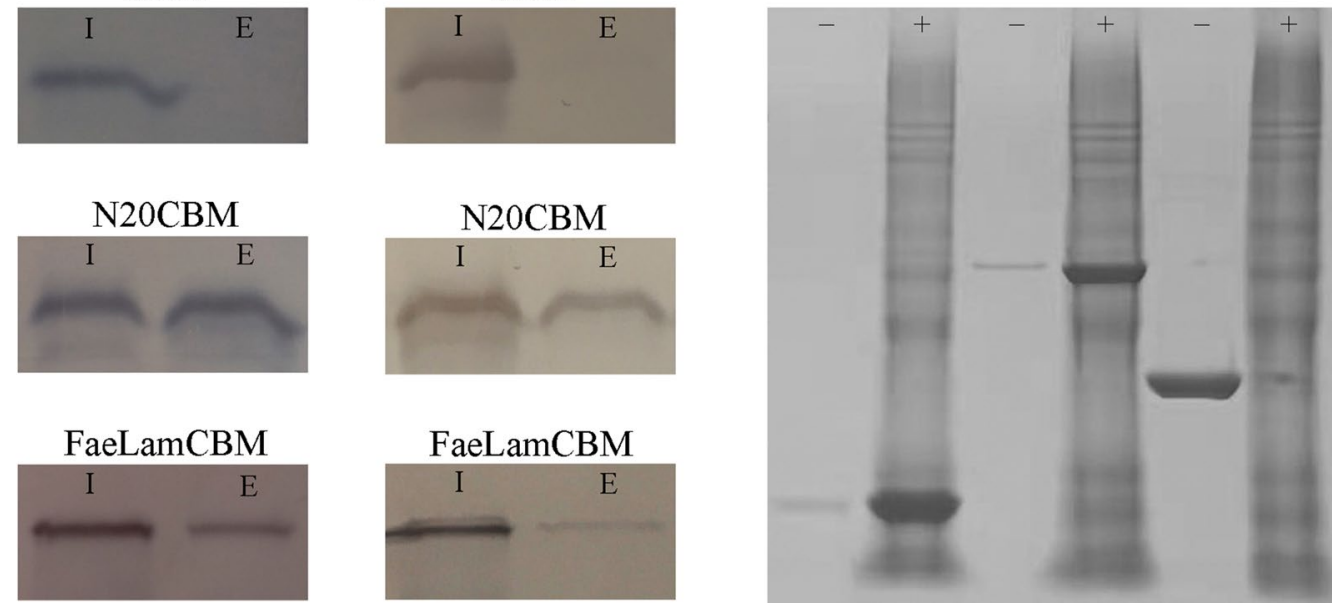

Fig. 5 The translocation and activity of heterologous protein fused with FaeLam or its N-terminus. A Western blot analysis of the subcellular localization of CBM, N20CBM and FaeLamCBM expressed in L. plantarum CGMCC6888; B Western blot analysis of the subcellular localization of CBM, N20CBM and FaeLamCBM expressed in E. coli DH5a; C SDS-PAGE analysis of the binding abilities of N20CBM, FaeLamCBM and FaeLam to de-starched wheat bran. I: intracellular fraction; E: extracellular fraction; -: unbound fraction; +: fraction bound to de-starched wheat bran

it was expressed without any additional sequences. This result also indicated that the detected extracellular proteins were not due to cell lysis. Therefore, the N20 could act as a signal peptide.

The N20CBM and FaeLamCBM were purified from the extracellular fraction of $E$. coli. The function of the CBM in the fusion proteins was identified by the binding abilities to the de-starched wheat bran. As shown in Fig. 5C, N20CBM and FaeLamCBM decreased from the unbound fraction, while prominent bands were detected in the bound fraction. Meanwhile, FaeLam which was used as a control still existed in the unbound fraction. These results indicated that CBM contained in the two fusion proteins had the ability to absorb the corresponding carbohydrate.

\section{Discussion}

Polysaccharide-bound hydroxycinnamic acids are widely present in cell walls [19]. Feruloyl esterases can cleave the ester bond between hydroxycinnamic acids and polysaccharide. The most reported feruloyl esterases are natural extracellular enzymes in their native organisms, which is beneficial for the enzymes to access their substrates [20]. However, it is still controversial that feruloyl esterases derived from Lactobacillus are located in intracellular or secreted out of cells. Feruloyl esterase has been identified in many Lactobacillus species, including L. gasseri [21], L. acidophilus [22], L. reuteri, L. casei [23], L. helveticus [24], L. johnsonii [7], L. fermentum [6] and L. crispatus [25]. Although bioinformatic analysis showed that no signal peptides were predicted in these Lactobacillus feruloyl esterases, the increase of free phenolic acids was detected in whole grain barley and oat groat by fermentation with Lactobacillus strains, indicating the secretory characteristics of their feruloyl estreases [23, 26]. Our results could shed light on the location of feruloyl esterases produced by Lactobacillus species. Furthermore, the L. plantarum with secreted feruloyl esterase might play roles in many applications. For example, it could be applied in ensiling of crop for improving fermentation quality and fibre digestibility of ensiled forages, and could be orally administered as a dietary supplement or functional food for increasing the intestinal feruloyl esterase activity to enhance the bioavailability of hydroxycinnamic acids, thus improving oxidative status [27, 28].

Our previous experiments showed that FaeLam could be secreted from strain E. coli BL21(DE3) [11]. In the present study, it could also be secreted into extracellular environment of $E$. coli $\mathrm{DH} 5 \alpha$, suggesting the secretion of FaeLam is not strain dependent. Since feruloyl esterase has significant potential applications in many industrial fields, the preparation of feruloyl esterase has drawn extensive attention in recent years. Therefore, these E. coli strains expressing the Lactobacillus feruloyl esterases are ideal hosts for feruloyl esterases production, although optimization is needed to improve the yields and productivity in the future. The secretion characteristic allows a simple process of product separation and purification. Furthermore, the recombinant $E$. coli, as well as the recombinant $L$. plantarum, can be directly used to produce value-added products from agricultural wastes, such as ferulic acid and vanillin [29, 30]. 
It was proven that the $\mathrm{N}$-terminal sequence of FaeLam played a crucial role in this secretion. Bioinformatic analysis revealed that the $\mathrm{N}$-terminus of FaeLam had good polarity and hydrophilicity (Additional file 1: Figure S2B, C). These characteristics were significantly different from the Sec signal peptide [31]. Thus, the feruloyl esterase could be classified as non-classically secreted protein, which seldom reported in lactic acid bacteria [32]. In our previous study, we found that ten feruloyl esterases produced by different lactic acid bacteria all could be secreted by $E$. coli, although with diverse secretion levels [11]. Sequence alignment showed that the N20 of these enzymes had a certain similarity, especially conserved in two leucine and a glycine (Additional file 1: Figure S6). Designing mutated enzymes at these sites will help to understand the role of different residues in secretion. In addition, although the sequence basis of the feruloyl esterase for secretion was determined in this work, it is necessary to further confirm whether there are related proteins required for the feruloyl esterase transportation in the strains, thus to fully understand the non-classical secretion pathway of feruloyl esterase [33].

We also demonstrated that FaeLam and its N-terminal 20 amino acid residues can be used as carriers for extracellular production of heterologous target proteins in $L$. plantarum CGMCC6888 and E. coli DH5 $\alpha$. To our best knowledges, this is the first report to show that a short peptide can serve as a signal peptide and guide heterologous protein out of the cells of both Gram-positive and Gram-negative bacteria. During the past years, numerous efforts have been made to explore the potential of $L$. plantarum and E.coli as cell factories for secreted expression recombinant proteins $[34,35]$. As for L. plantarum, heterologous protein secretion dependent on typical Sec pathway is usually very inefficient, and cytoplasmic proteins are usually not translocated across the cell membrane with the aid of signal peptides [36, 37]. These factors limit the use of $L$. plantarum as a cell factory and highlight the importance of exploring new transport routes for secreted heterologous proteins. E. coli, a Gram-negative bacterium, has two membranes, which makes it even harder to secrete heterologous proteins than in Gram-positive bacterium. Secretory production of proteins in $E$. coli has many possible applications, and will simplify the protein production process and decrease production costs of enzymes [38, 39]. Therefore, the $\mathrm{N}$-terminus of FaeLam provides a new tool for the secretion of recombinant proteins in L. plantarum and E. coli.

\section{Conclusions}

The feruloyl esterase derived from $L$. amylovorus could be secreted to the extracellular of L. plantarum CGMCC6888 and E. coli DH5 $\alpha$. We proved the
$\mathrm{N}$-terminus of FaeLam played the important role in its secretion. Although further studies should be conducted for fully understanding the secretion mechanism, the present study provided alternative strains for the production of feruloyl esterase and ferulic acid. In addition, the $\mathrm{N}$-terminus of FaeLam also could be employed as carriers for extracellular production of recombinant proteins. L. plantarum and E. coli are important model species in molecular biology research and often used as expression hosts for heterologous proteins. Therefore, a new method was established for protein secretion in L. plantarum and $E$. coli through fusion the targeted protein to N20 of FaeLam.

\section{Material and methods}

Bacterial strains, culture conditions, plasmids and chemicals

The strains L. amylovorus CGMCC11056 and L. plantarum CGMCC6888 were cultured statically at $37{ }^{\circ} \mathrm{C}$ in MRS (de Man, Rogosa and Sharpe) broth (Oxoid, Basingstoke, UK). E. coli DH5 $\alpha$ was cultured in LB (LuriaBertani) medium at $37{ }^{\circ} \mathrm{C}$ aerobically. If necessary, chloramphenicol was added with a final concentration of $10 \mu \mathrm{g} / \mathrm{mL}$ for $E$. coli or $5 \mu \mathrm{g} / \mathrm{mL}$ for L. plantarum. The plasmids used in this study were listed in Table 2. Methyl ferulate and ethyl ferulate used as substrates for enzyme assays of feruloyl esterase, were purchased from Sigma Chemicals Industries Co., Ltd. (SanFrancisco, USA). All PCR reactions were performed with Ex Taq polymerase (TaKaRa, Tokyo, Japan). Restriction enzymes and $\mathrm{T}_{4}$ DNA ligase were also purchased from TaKaRa Biotechnology Co., Ltd. (Tokyo, Japan). DNA extraction kit, agarose gel DNA purification kit, and cycle pure kit were obtained from Omega Bio-tek (Atlanta, USA).

\section{DNA manipulations}

To express proteins in both of L. plantarum and E. coli, a shuttle vector pLP3804 was constructed. The primers used in this study were listed in Additional file 1: Table $\mathrm{S} 1$. The promoter $\left(\mathrm{P}_{\text {tuf }}\right)$ of the putative elongation factor and the terminator (Ter) were amplified with the genomic DNA of L. plantarum CGMCC6888 and Lactococcus lactic MG1363 as templates, respectively [14]. The repA of pD403 and the replicon of pUC19 were responsible for replication of the vector in L. plantarum and $E$. coli [40]. The chloramphenicol resistance gene derived from pNZ8148 was used as the selection marker [41]. The multiple cloning sites were included in the synthetic primers.

The faeLam gene encoding FaeLam was amplified from L. amylovorus CGMCC11056 using the primers FaeLamF and FaeLam-R-His ${ }_{6}$ (Table 3) [17]. Then the purified PCR product was digested with the restriction enzymes 
Table 2 The plasmids used in this study

\begin{tabular}{|c|c|c|}
\hline Plasmids & Description & Source \\
\hline pUC19 & Ampre ori & Novagen \\
\hline pD403 & $2.8 \mathrm{~kb}$ cryptic plasmid of L. planturum D403 & Our laboratory \\
\hline pET22b-FaeLam & Amp', pET-22b vector ligated with faeLam gene & Our laboratory \\
\hline pNZ8148 & $\mathrm{Cm}^{r}$ & $\begin{array}{l}\text { Mierau and } \\
\text { Kleerebezem } \\
\text { [41] }\end{array}$ \\
\hline pMN402 & Derivation of gfp gene & Scholz et al. [15] \\
\hline pLP3804 & $\mathrm{Cm}^{r}$, ori from pUC19, ori and repA from pD403, promoter $\mathrm{P}_{\text {tuf }}$ & This study \\
\hline pLP-GFP & pLP3804, gfp gene under promoter $P_{\text {tuf }}$ & This study \\
\hline pLP-FaeLam & pLP3804, faeLam gene under promoter $P_{\text {tuf }}$ & This study \\
\hline pLP- $\triangle 20 F a e L a m$ & pLP3804, faelam gene that truncated 60 bp at $5^{\prime}$ region & This study \\
\hline pLP-FaeLam ${ }^{\text {S106A }}$ & pLP3804, the mutated faeLam gene under promoter $P_{\text {tuf }}$ & This study \\
\hline pLP-CBM & pLP3804, cbm from Paenibacillus panacisoli under promoter $P_{\text {tuf }}$ & This study \\
\hline PLP-N20CBM & pLP3804, n20 gene fusion with cbm from Paenibacillus panacisoli & This study \\
\hline pLP-FaeLamCBM & pLP3804, faeLam gene fusion with cbm from Paenibacillus panacisoli & This study \\
\hline
\end{tabular}

Table 3 The primers used in this study

\begin{tabular}{|c|c|c|}
\hline Primer & Sequence $\left(5^{\prime}-3^{\prime}\right)$ & Restriction sites \\
\hline GFP-F & GCGCATATGAGCAAAGGAGAAGAAC & Ndel \\
\hline GFP-R & ATAGGATCCTTAGTATAGCTCATCCATG & $\mathrm{BamHI}$ \\
\hline FaeLam-F & TATACATATGTCCCGCATTACAATTG & Ndel \\
\hline FaeLam-R-His 6 & GCGCTCGAGTTAGTGGTGGTGGTGGTGGTGGAATAATGGTTTTAAAAATT & Xhol \\
\hline S106A-F & GTCATGCTCAAGGCGGCG & \\
\hline S106A-R & CGCCGCCTTGAGCATGACCTACTAAAAAGATATTGC & \\
\hline FaeLam $\triangle 20-F$ & ATACATATGTTTGGCGAAATTTATGACATG & Ndel \\
\hline CBM-F1 & ACACTTACGATTGGAGGCAG & \\
\hline CBM-F2 & ATACATATGACACTTACGATTGGAGGCAG & Ndel \\
\hline $\mathrm{CBM}-\mathrm{R}-\mathrm{His}_{6}$ & ATACTCGAGTTAGTGATGATGATGATGATGATGGATGTCCAAATAGTC & Xhol \\
\hline N20CBM-R & GCCTCCAATCGTAAGTGTAGGCTCTTCACGATCTCC & \\
\hline FaeLamCBM-R & GCCTCCAATCGTAAGTGTCTAGAATAATGGTTTT & \\
\hline
\end{tabular}

NdeI and XhoI and ligated into the expression vector pLP3804 restricted with the same enzymes, obtaining the recombinant plasmid pLP-FaeLam. The mutant plasmid pLP-FaeLam ${ }^{\text {S106A }}$ was generated by utilizing the overlap extending methodology with the primers S106A-F and S106A-R, and confirmed by hydrolytic activity determination. The DNA sequence encoding FaeLam with 20 amino acid residues deletion at the $\mathrm{N}$-terminus was amplified from the plasmids pLP-FaeLam with primers FaeLam $\Delta 20-\mathrm{F}$ and FaeLam-R-His ${ }_{6}$, generating the plasmid pLP- $\Delta 20$ FaeLam.

To fuse the protein CBM derived from P. panacisoli to the N20 amino acid residues of FaeLam [18], primer sets FaeLam-F/N20CBM-R and CBM-F1/CBM-R-His ${ }_{6}$ were used to amplify the N20 and CBM sequences, and then these two fragments were fused by the overlap extending methodology. The resulting product was digested with the restriction enzymes NdeI and XhoI and ligated into the corresponding sites of vector pLP3804 to obtain the plasmid pLP-N20CBM. The same method was used to fuse genes faeLam and $c b m$, and recombinant plasmid pLP-FaeLamCBM was obtained. Furthermore, pLP-CBM was constructed as a control by using primers CBM-F2 and $\mathrm{CBM}-\mathrm{R}-\mathrm{His}_{6}$. All the resulting plasmids were confirmed by DNA sequencing.

\section{Transformation}

To prepare the $L$. plantarum competent cells, an overnight culture was transferred into $5 \mathrm{~mL}$ of SGMRS (MRS added $0.75 \mathrm{M}$ sorbitol and $1 \%$ glycine) as the inoculation 
volume of $1 \%$, and incubated at $37^{\circ} \mathrm{C}$. When the $\mathrm{OD}_{600}$ reached 0.6 , cultures were centrifuged at $4000 \times g, 4{ }^{\circ} \mathrm{C}$ for $5 \mathrm{~min}$. The sedimented cells were washed with electroporation buffer $\left(0.05 \mathrm{M}\right.$ sucrose, $\left.1 \mathrm{mM} \mathrm{MgCl}_{2}\right)$ three times and then resuspended in $40 \mu \mathrm{L}$ electroporation buffer. For electro-transformation of $L$. plantarum, the $40 \mu \mathrm{L}$ fresh prepared competent cells were gently mixed with $500 \mathrm{ng}$ DNA and left on ice for $5 \mathrm{~min}$. The mixture was transferred into a pre-cooled cuvette with a gap of $0.2 \mathrm{~cm}$ (Bio-Rad, California, USA) and electroporated by a pulse of $2000 \mathrm{~V}$ at $25 \mu \mathrm{F}$ (Gene Pulser, Bio-Rad, California, USA). After electroporation, $960 \mu \mathrm{L}$ of SMRS broth (MRS with $0.75 \mathrm{M}$ Sorbitol and $10 \mathrm{mM} \mathrm{CaCl}_{2}$ ) was immediately added and the cells were incubated at $37^{\circ} \mathrm{C}$ for $2 \mathrm{~h}$. Competent cells of E. coli DH5 $\alpha$ were prepared and transformed with plasmid DNA as described in the previous study [42]. To select the transformants, the culture was plated on LB or MRS plates containing chloramphenicol with appropriate concentrations.

\section{Fluorescence assay}

Recombinant strains harboring the pLP3804 and pLPGFP were cultured and taken out after $12 \mathrm{~h}$ for the fluorescence measurement. The cells were collected by centrifugation at $10,000 \times g$ for $5 \mathrm{~min}$, and then washed twice using PBS buffer $(137 \mathrm{mM} \mathrm{NaCl}, 2.7 \mathrm{mM} \mathrm{KCl}$, $10 \mathrm{mM} \mathrm{Na}{ }_{2} \mathrm{HPO}_{4}, 2 \mathrm{mM} \mathrm{KH} \mathrm{PO}_{4}$, pH 7.4). After being resuspend in an equal volume of buffer, $200 \mu \mathrm{L}$ of bacterial suspension was transferred into a 96-well plate. The fluorescence was detected by a Multi-Detection Microplate Reader with the excitation at $485 \mathrm{~nm}$ and the emission at $528 \mathrm{~nm}$.

\section{Feruloyl esterase activity assay}

The plate-based assay to preliminarily determine feruloyl esterase activity was as follows. Ethyl ferulate with a concentration of $6.7 \mathrm{mM}$ was added in the MRS and LB plates as substrate. Strains or samples were loaded into the plate, and then incubated at $37{ }^{\circ} \mathrm{C}$. The hydrolytic zone was examined and photographed. Furthermore, the $\rho N P F$ was used as substrate to quantitatively determine the feruloyl esterase activity according to our previous work [11].

High performance liquid chromatography (HPLC) method was also employed to detect the hydrolytic activity of feruloyl esterase. The enzyme reaction was carried out in $1 \mathrm{~mL}$ of intracellular or extracellular fraction containing $1 \mathrm{mM}$ methyl ferulate. After $12 \mathrm{~h}$ incubation at $37{ }^{\circ} \mathrm{C}, 1 \mathrm{~mL} 50 \%$ acetate (v/v) was added to terminate the reaction. The HPLC was equipped with an XBridge BEH300 C18 reverse phase column $(150 \mathrm{~mm} \times 4.6 \mathrm{~mm}$; Waters, Milford, USA) at a flow rate of $1.0 \mathrm{~mL} / \mathrm{min}$. The mobile phase was composed of solvent A (methanol) and solvent B (water and acetic acid, 99:1, v/v) at a ratio of $1: 1$, and the column eluent was monitored at the $A_{320}$ with temperature of $35^{\circ} \mathrm{C}$ [17].

\section{Functional analysis of the carbohydrate binding module}

To verify the function of CBM contained in the fusion proteins, their binding ability to de-starched wheat bran was detected. The cultures of E. coli secreting N20CBM and FaeLamCBM were centrifugated at $10,000 \times g, 4{ }^{\circ} \mathrm{C}$ for $10 \mathrm{~min}$. The supernatant was collected and filtered by $0.22-\mu \mathrm{m}$ filter. Extracellular proteins were concentrated using ultrafiltration tubes (Millipore, Massachusetts, USA) and dialyzed against PBS buffer. Then, the target proteins were obtained by the affinity chromatography of Ni-TED column (GE Healthcare, Stockholm, Sweden). Subsequently, the purified proteins were mixed with destarched wheat bran at $5 \%(\mathrm{w} / \mathrm{v})$ final concentration and kept at $4{ }^{\circ} \mathrm{C}$ for $3 \mathrm{~h}$. After centrifuged at $10,000 \times \mathrm{g}, 4^{\circ} \mathrm{C}$ for $10 \mathrm{~min}$, the supernatant was collected as unbound fraction. The pellets were washed with PBS buffer, and then the bound fraction was eluted by washing with $1 \times$ SDS-PAGE loading buffer.

\section{SDS-PAGE and western blot analysis}

Sodium dodecyl sulfate-polyacrylamide gel electrophoresis (SDS-PAGE) with a $5 \%$ stacking gel and a $12 \%$ separating gel was used to separate the protein bands, and then protein bands in the gel were visualized by Coomassie brilliant blue staining.

To conduct western blot analysis, a PVDF membrane was used to electro-transfer the separated proteins in SDS-PAGE gel at $200 \mathrm{~mA}$ with appropriate time, and then blocked with $5 \%$ non-fat milk for $1 \mathrm{~h}$. Considering that the $\mathrm{C}$-terminus of target proteins was labeled a $6 \times$ His tag, the primary antibody anti-six-histidine mouse IgG (1:1,000 dilution; Novagen, USA) was used to incubate the membrane for $12 \mathrm{~h}$. Subsequently, the membrane was washed three times with TBST buffer $(20 \mathrm{mM}$ Tris- $\mathrm{HCl}, 500 \mathrm{mM} \mathrm{NaCl}, 0.01 \%$ Tween-20, pH 7.4). The secondary antibody horseradish-peroxidase (HRP)labeled goat anti-mouse IgG (1:300 dilution; Solarbio, China) was used to incubate the membrane for $1 \mathrm{~h}$. The positive protein bands in the PVDF membrane were visualized using a HRP-DAB kit (Tiangen, Beijing, China).

\section{Cell fractionation}

For enzymatic analysis, intracellular and extracellular were prepared as follows. The culture broth after $12 \mathrm{~h}$ cultivation was centrifuged at $10,000 \times g, 4{ }^{\circ} \mathrm{C}$ for $10 \mathrm{~min}$, and the supernatant was collected and filtered by 0.22 $\mu \mathrm{m}$ filter as the extracellular fraction. The centrifuged cells were washed and resuspended in an equal volume of PBS buffer, and then disrupted by a Precellys 24 (Bertin, 
Paris, France). After centrifugation at $10,000 \times g, 4{ }^{\circ} \mathrm{C}$ for $10 \mathrm{~min}$, the supernatant was collected and filtered as intracellular fraction.

For western blot analysis, cell fractionation of L. plantarum and E. coli was performed as follows. Five milliliter of L. plantarum CGMCC6888 culture broth after $12 \mathrm{~h}$ cultivation was centrifuged at $10,000 \times g, 4{ }^{\circ} \mathrm{C}$ for $10 \mathrm{~min}$, the resulting supernatant was filtered through a $0.22-\mu \mathrm{m}$ filter and ice-cold trichloroacetic acid was added as the final concentration of $10 \%(\mathrm{v} / \mathrm{v})$. After incubation on ice for $1 \mathrm{~h}$, the supernatant was centrifuged at $12,000 \times g, 4{ }^{\circ} \mathrm{C}$ for $15 \mathrm{~min}$, and the precipitate was washed twice with ice-cold acetone, and then it was air-dried and dissolved in $100 \mu \mathrm{L}$ PBS buffer to obtain the extracellular fraction. The centrifuged cells were washed and resuspended in $1 \mathrm{~mL}$ PBS buffer, and then disrupted by a Precellys 24 (Bertin, Paris, France). After centrifugation at $10,000 \times g, 4{ }^{\circ} \mathrm{C}$ for $10 \mathrm{~min}$, the supernatant was collected as intracellular fraction. One milliliter of E. coli DH5 $\alpha$ culture broth after $12 \mathrm{~h}$ cultivation was centrifuged at $10,000 \times g, 4{ }^{\circ} \mathrm{C}$ for $10 \mathrm{~min}$, and the supernatant was collected and filtered by 0.22 $\mu \mathrm{m}$ filter as the extracellular fraction. The preparation of $E$. coli DH5 $\alpha$ intracellular fraction was same as that of L. plantarum CGMCC6888.

\author{
Abbreviations \\ N20: 20 Amino acids of N-terminus; GRAS: Generally recognized as safe; \\ GFP: Green fluorescence protein; CBM: Carbohydrate binding module; \\ IPTG: Isopropyl- $\beta$-D thiogalactopyranoside; SDS-PAGE: Sodium dodecylsul- \\ phate polyacrylamide gel electrophoresis; HPLC: High performance liquid \\ chromatography.
}

\section{Supplementary Information}

The online version contains supplementary material available at https://doi. org/10.1186/s12934-021-01645-9.

Additional file 1: Figure S1. SDS-PAGE analysis of the expression of green fluorescence protein in L. plantarum CGMCC6888 and E. coli DH5a. Figure S2. The signal peptide prediction of FaeLam by using SignalP-5.0 server (A). The polarity (B) and hydrophilicity (C) prediction of FaeLam by using Protscale. Figure S3. HPLC analysis of methyl ferulate degradation by intracellular (A) or extracellular (B) fractions of L. plantarum CGMCC6888 harboring pLP-FaeLam. The corresponding heat-inactivated intracellular (C) or extracellular (D) fractions were also conducted as controls. The peaks for ferulic acid and methyl ferulate were detected at $2.5 \mathrm{~min}$ and $4 \mathrm{~min}$, respectively. Figure S4. Feruloyl esterase activity analysis of intracellular (A) or extracellular (B) fractions of E. coli DH5a harboring pLPFaeLam. Figure S5. Analysis of the N-terminal sequence of extracellular FaeLam. Figure $\mathbf{S 6}$. Sequence alignment of feruloyl esterases from different Lactobacillus strains by using Clustal Omega. The 20 amino acid of $\mathrm{N}$-terminus was circled. Figure S7. The full gels of the western blot results. Targeted bands were circled by red square. Table S1. Primers used in the vector construction of pLP3804.

\section{Acknowledgements}

Not applicable.

\section{Authors' contributions}

ZSX and JK conceived and designed the experiments, ZSX and RLZ carried out the experimental work. ZSX, TW and JK wrote and revised the manuscript. All authors read and approved the final manuscript.

\section{Funding}

This work was financially supported by National Natural Science Foundation of China (31901665), Public Service Sectors (Agriculture) Special and Scientific Research Projects (201503134), Key Research and Development Program of Shandong Province (2020CXGC010602), Science and Technology Support Plan for Young People in Colleges and Universities of Shandong Province (2020KJE005), Young Doctor Cooperative Project of Qilu University of Technology, Shandong Academy of Sciences (2019BSHZ0020), and the Foundation (ZZ20200108) of State Key Laboratory of Biobased Material and Green Papermaking, Qilu University of Technology, Shandong Academy of Sciences.

\section{Availability of data and materials}

All data generated or analysed during this study are included in this published article.

\section{Declarations}

Ethics approval and consent to participate

Not applicable.

\section{Consent for publication}

Not applicable.

\section{Competing interests}

The authors declare that they have no competing interests.

\section{Author details}

${ }^{1}$ State Key Laboratory of Biobased Material and Green Papermaking, Qilu University of Technology, Shandong Academy of Science, No.3501, Daxue Road, Jinan 250353, People's Republic of China. ${ }^{2}$ School of Bioengineering, Qilu University of Technology, Shandong Academy of Science, Jinan 250353, People's Republic of China. ${ }^{3}$ State Key Laboratory of Microbial Technology, Shandong University, No.72, Binhai Road, Qingdao 266237, Shandong, People's Republic of China.

Received: 21 October 2020 Accepted: 25 July 2021

Published online: 03 August 2021

\section{References}

1. Crepin VF, Faulds CB, Connerton IF. Functional classification of the microbial feruloyl esterases. Appl Microbiol Biotechnol. 2004;63:647-52.

2. Fazary AE, Ju YH. Feruloyl esterases as biotechnological tools: current and future perspectives. Acta Biochem Biophys Sin. 2007;39:811-28.

3. Ou SY, Kwok KC. Ferulic acid: pharmaceutical functions, preparation and applications in foods. J Sci Food Agric. 2004;84:1261-9.

4. Topakas E, Stamatic H, Biely P, Kekos D, Macris BJ, Christakopulos P. Purification and characterization of a feruloyl esterase from Fusarium oxysporum catalyzing esterification of phenolic acid in ternary waterorganic solvent mixtures. J Biotechnol. 2003;102:33-44.

5. Fritsch C, Jänsch A, Ehrmann MA, Toelstede S, Vogel RF. Characterization of cinnamoyl esterases from different Lactobacilli and Bifidobacteria. Curr Microbiol. 2016;74:247-56.

6. Liu S, Bischoff KM, Anderson AM, Rich JO. Novel feruloyl esterase from Lactobacillus fermentum NRRL B-1932 and analysis of the recombinant enzyme produced in Escherichia coli. Appl Environ Microbiol. 2016;82:5068-76.

7. Lai KK, Lorca GL, Gonzalez CF. Biochemical properties of two cinnamoyl esterases purified from a Lactobacillus johnsonii strain isolated from stool samples of diabetes-resistant rats. Appl Environ Microbiol. 2009;75:5018-24. 
8. Ferrer-Miralles N, Domingo-Espin J, Corchero JL, Vazquez E, Villaverde A. Microbial factories for recombinant pharmaceuticals. Microb Cell Fact. 2009:8:17.

9. Jong WS, Sauri A, Luirink J. Extracellular production of recombinant proteins using bacterial autotransporters. Curr Opin Biotechnol. 2010;21:646-52.

10. Desvaux M, Hebraud M, Talon R, Henderson IR. Secretion and subcellular localizations of bacterial proteins: a semantic awareness issue. Trends Microbiol. 2009:17:139-45.

11. Xu Z, Kong J, Zhang S, Wang T, Liu X. Comparison of enzyme secretion and ferulic acid production by Escherichia coli expressing different Lactobacillus feruloyl esterases. Front Microbiol. 2020;11:568716.

12. Su L, Woodard RW, Chen J, Wu J. Extracellular location of Thermobifida fusca cutinase expressed in Escherichia coli BL21(DE3) without mediation of a signal peptide. Appl Environ Microbiol. 2013;79:4192-8.

13. Gao D, Wang S, Li H, Yu H, Qi Q. Identification of a heterologous cellulase and its $\mathrm{N}$-terminus that can guide recombinant proteins out of Escherichia coli. Microb Cell Fact. 2015;14:49.

14. Tauer C, Heinl S, Egger E, Heiss S, Grabherr R. Tuning constitutive recombinant gene expression in Lactobacillus plantarum. Microb Cell Fact. 2014;13:150.

15. Scholz O, Thiel A, Hillen W, Niederweis M. Quantitative analysis of gene expression with an improved green fluorescent protein. Eur J Biochem. 2000;267:1565-70.

16. Landete JM, Langa S, Revilla C, Margolles A, Medina M, Arqués JL. Use of anaerobic green fluorescent protein versus green fluorescent protein as reporter in lactic acid bacteria. Appl Microbiol Biotechnol. 2015;99:6865-77.

17. Xu Z, He H, Zhang S, Guo T, Kong J. Characterization of feruloyl esterases produced by the four Lactobacillus species: L. amylovorus, L. acidophilus, $L$ farciminis and $L$. fermentum, isolated from ensiled corn stover. Front Microbiol. 2017:8:941.

18. Xu Z, Zhang S, Mu Y, Kong J. Paenibacillus panacisoli enhances growth of Lactobacillus spp. by producing xylooligosaccharides in corn stover ensilages. Carbohydr Polym. 2018;184:435-44.

19. Akin DE. Plant cell wall aromatics: influence on degradation of biomass. Biofuels Bioprod Bioref. 2008;2:303-86.

20. Dilokpimol A, Mäkelä MR, Mansouri S, Belova O, Waterstraat M, Bunzel $M$, de Vries RP, Hildén KS. Expanding the feruloyl esterase gene family of Aspergillus niger by characterization of a feruloyl esterase. FaeC N Biotechnol. 2017;37:200-9.

21. Couteau D, McCartney AL, Gibson GR, Williamson G, Faulds CB. Isolation and characterization of human colonic bacteria able to hydrolyse chlorogenic acid. J Appl Microbiol. 2001;90:873-81.

22. Wang X, Geng X, Egashira Y, Sanada H. Purification and characterization of a feruloyl esterase from the intestinal bacterium Lactobacillus acidophilus. Appl Environ Microbiol. 2004;70:2367-72.

23. Hole AS, Rud I, Grimmer S, Sigl S, Narvhus J, Sahlstrøm S. Improved bioavailability of dietary phenolic acids in whole grain barley and oat groat following fermentation with probiotic Lactobacillus acidophilus, Lactobacillus johnsonii, and Lactobacillus reuteri. J Agric Food Chem. 2012;60:6369-75.

24. Guglielmetti S, de Noni I, Caracciolo F, Molinari F, Parini C, Mora D. Bacterial cinnamoyl esterase activity screening for the production of a novel functional food product. Appl Environ Microbiol. 2008;74:1284-8.

25. Xu Z, Wang T, Zhang S. Extracellular secretion of feruloyl esterase derived from Lactobacillus crispatus in Escherichia coli and its application for ferulic acid production. Bioresour Technol. 2019;288:12152.

26. Esteban-Torres M, Reverón I, Mancheño JM, de Las RB, Muñoz R. Characterization of a feruloyl esterase from Lactobacillus plantarum. Appl Environ Microbiol. 2013;79:5130-6.
27. Ding ZT, Xu DM, Bai J, Li FH, Adesogan AT, Zhang P, Yuan XJ, Guo XS. Characterization and identification of ferulic acid esterase-producing Lactobacillus species isolated from Elymus nutans silage and their application in ensiled alfalfa. J Appl Microbiol. 2019;127:985-95.

28. Mukdsi MC, Cano MP, González SN, Medina RB. Administration of Lactobacillus fermentum CRL1446 increases intestinal feruloyl esterase activity in mice. Lett Appl Microbiol. 2012;54:18-25.

29. Salgado JM, Rodríguez-Solana R, Curiel JA, de las Rivas B, Muñoz R, Domínguez JM. Production of vinyl derivatives from alkaline hydrolysates of corn cobs by recombinant Escherichia coli containing the phenolic acid decarboxylase from Lactobacillus plantarum CECT 748T. Bioresour Technol. 2012;117:274-85.

30. Chakraborty D, Gupta G, Kaur B. Metabolic engineering of E. coli top 10 for production of vanillin through FA catabolic pathway and bioprocess optimization using RSM. Protein Expr Purif. 2016;128:123-33.

31. Rapoport TA, Li L, Park E. Structural and mechanistic insights into protein translocation. Annu Rev Cell Dev Biol. 2017;33:369-90.

32. Saad N, Urdaci M, Vignoles C, Chaignepain S, Tallon R, Schmitter JM, Bressollier P. Lactobacillus plantarum 299v surface-bound GAPDH: a new insight into enzyme cell walls location. J Microbiol Biotechnol. 2009;19:1635-43

33. Wang G, Chen H, Xia Y, Cui J, Gu Z, Song Y, Chen YQ, Zhang H, Chen W. How are the non-classically secreted bacterial proteins released into the extracellular milieu? Curr Microbiol. 2013;67:688-95.

34. Choi JH, Lee SY. Secretory and extracellular production of recombinant proteins using Escherichia coli. Appl Microbiol Biotechnol. 2004:64:625-35.

35. Mathiesen G, Sveen A, Brurberg MB, Fredriksen L, Axelsson L, Eijsink VG. Genome-wide analysis of signal peptide functionality in Lactobacillus plantarum WCFS1. BMC Genomics. 2009;10:425.

36. van Wely KHM, Swaving J, Freudl R, Driessen AJM. Translocation of proteins across the cell envelope of Gram-positive bacteria. FEMS Microbiol Rev. 2001;25:437-54.

37. Hu S, Kong J, Sun Z, Han L, Kong W, Yang P. Heterologous protein display on the cell surface of lactic acid bacteria mediated by the S-layer protein Microb Cell Fact. 2011;10:86

38. Shin HD, Chen RR. Extracellular recombinant protein production from an Escherichia coli Ipp deletion mutant. Biotechnol Bioeng. 2008;101:1288-96.

39. Robbens J, Raeymaekers A, Steidler L, Fiers W, Remaut E. Production of soluble and active recombinant murine interleukin-2 in Escherichia coli: high level expression, Kil-induced release, and purification. Protein Expr Purif. 1995;6:481-6.

40. Sun Z, Kong J, Kong W. Characterization of a cryptic plasmid pD403 from Lactobacillus plantarum and construction of shuttle vectors based on its replicon. Mol Biotechnol. 2010;45:24-33.

41. Mierau I, Kleerebezem M. 10 years of the nisin controlled gene expression system (NICE) in Lactococcus. Appl Microbiol Biotechnol. 2005;68:705-17.

42. Cohen SN, Chang AC, Hsu L. Nonchromosomal antibiotic resistance in bacteria: genetic transformation of Escherichia coli by R-factor DNA. Proc Natl Acad Sci USA. 1972;69:2110-4.

\section{Publisher's Note}

Springer Nature remains neutral with regard to jurisdictional claims in published maps and institutional affiliations. 